International Mathematical Forum, Vol. 9, 2014, no. 20, 981 - 986 HIKARI Ltd, www.m-hikari.com http://dx.doi.org/10.12988/imf.2014.4348

\title{
Representation Type under Excellent Extensions
}

\author{
Juxiang Sun
}

\author{
School of Mathematics and Information Science \\ Shangqiu Normal University, Shangqiu, 476000, China
}

Copyright (C) 2014 Juxiang Sun. This is an open access article distributed under the Creative Commons Attribution License, which permits unrestricted use, distribution, and reproduction in any medium, provided the original work is properly cited.

\begin{abstract}
In this paper, we prove that representation type of finite dimensional algebras over an algebraically closed field is an invariant under excellent extensions.
\end{abstract}

Mathematics Subject Classification: 16K20, 16G60

Keywords: excellent extension; tame algebra; representation type

\section{Introduction}

In studying the algebraic structure of group rings, Passman in [9] introduced the notion of the excellent extensions of rings( the name comes from [1]). Such extensions of rings are vital since they include two important classes of extensions of rings: finite matrix rings and skew group rings $A G$, where the finite group $G$ satisfies the condition $|G|^{-1} \in A$. Many authors have studied the invariant properties of rings under excellent extensions $([1,5,6,9,10,11,12])$.

It is well known that determining the representation type of algebras is fundamental and important in representation theory of finite dimensional algebras. Let $k$ be an algebraically closed field. Recall that a finite dimensional $k$-algebra $A$ is said to be of finite representation type or a representation finite algebra, if there exist only finitely many isomorphism classes of finitely 
generated indecomposable $A$-modules. Recall that the a finite dimensional $k$-algebra $A$ is said to be of tame type or a tame algebra if $A$ is not of finite representation type, whereas for any dimension $d>0$, there are a finite number of $k[T]-A$-bimodules $\left\{M_{i}: i=1,2, \cdots, n\right\}$, which are free as left $k[T]$-modules such that all but a finite number of indecomposable $A$-modules of dimension $d$ are isomorphic to $k[T] /(T-\lambda) \otimes_{k[T]} M_{i}$ for $\lambda \in k$. And, a finite dimensional $k$-algebra $A$ is said to be of wild type or a wild algebra if there is a finitely generated $k\langle X, Y>-A$-bimodule $N$, which is free as

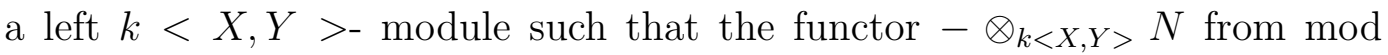
$k\langle X, Y\rangle$ to $\bmod A$ preserves indecomposability and reflects isomorphisms. The famous tame-and-wild theorem of Drozd's in [3, 4] states that a finite dimensional k-algebra, which is not of finite representation type, is either of tame type or of wild type, and not both. Therefore, it gives the classification of finite dimensional algebras over an algebraically closed field .

Let $B \geq A$ be an excellent extension of a finite dimensional $k$-module $A$. $B$ is of finite representation type if and only if so is $A$ by [6]. On the other hand, $A G$ ( $G$ is a finite group such that $|G|^{-1} \in A$, where $A$ is a finite dimensional $k$-algebra) and $A$ have the same representation type by [8]. And S. Kasjan in [7] showed that $A$ is a tame algebra if and only if so is $A \otimes_{k} F$, where $F$ is a separable field over $k$. Based on these facts, it is natural to ask the following question:

Question: Let $B \geq A$ be an excellent extension of an finite dimensional $k$-algebra, do $A$ and $B$ have the same representation type?

In this paper, we will study the invariance of the representation type of finite dimensional $k$-algebras under excellent extensions and give a correct answer to this question. This paper is organized as follows.

In Section 2, we give some notations in our terminology and some preliminary results which are often used in this paper. In Section 3, we prove the following

Theorem 1.1 Let $B$ be an excellent extension of a finite dimensional $k$ algebra $A$, then $A$ and $B$ have the same representation type.

\section{Preliminaries}

Throughout this paper, All modules are finitely generated right modules unless stated otherwise. 
We begin with the definition of excellent extensions of finite dimensional $k$-algebras.

Definition 2.1. Let $A$ be a subalgebra of a finite dimensional $k$-algebra $B$, such that $A$ and $B$ have the same identity. Then $B$ is called an algebraic extension of $A$, and denoted by $A \leq B$. An algebraic extension $A \leq B$ is called an excellent extension, if

(1) $A \leq B$ is right $A$-projective ([9], p.273), that is, if $N_{B}$ is a submodule of $M_{B}$ and if $N_{A}$ is a direct summand of $M_{A}$, denoted by $N_{A} \mid M_{A}$, then $N_{B} \mid M_{B}$.

(2) $B$ is a free normalizing extension of $A$ with a basis that includes 1 ; that $i s$, there is a finite set $\left\{b_{1}, b_{2}, \cdots, b_{n}\right\} \in B$ such that $b_{1}=1, B=b_{1} A+\cdots+b_{n} A$ and $b_{i} A=A b_{i}$, for each $i$, and $B$ is free with basis $\left\{b_{1}, b_{2}, \cdots, b_{n}\right\} \in B$ as both a right and left $A$-module.

Lemma 2.2. (See [[9], p.275, Lemma 2.3].) Let $A$ be a finite dimensional $k$-algebra, and let $F$ be a finite separable field extension of $k$. Then $A \otimes_{k} F$ is an excellent extension of $A$.

Lemma 2.3.(See[[6], Theorem 3.8].) Let $B$ be an excellent extension of a finite dimensional $k$-algebra $A$. If $A$ is of finite representation type if and only if so is $B$.

Lemma 2.4. ( See [[11], Lemma 1.1].) Let $A \leq B$ be an algebraic extension such that $B$ is right $A$-projective. Then we have $M_{B} \mid\left(M \otimes_{A} B\right)_{B}$ for any $B$ module.

Let $A$ be a finite dimensional $k$-algebra. A category is called generic category, denoted by $G C(A)$, if its objects are all $k[T]-A$-bimodules which are free as left $k[T]$-modules, and its morphisms are all $k[T]-A$-morphisms. It is closely related with tame algebras and thus with generic modules [2]. The following lemma are taken from [8], which plays an important role in this paper.

Lemma 2.5. Let $X \in G C(A)$. Then $X$ is indecomposable in $G C(A)$ if and only if $k[T] /(T-\lambda) \otimes_{k[T]} X$ is indecomposable as a $k[T] /(T-\lambda)$-A-bimodule for $\lambda \in k$.

\section{Representation type}

By the definition of excellent extensions, it is easy to prove the following

Lemma 3.1. Let $B \geq A$ be an excellent extension. If $A$ is a finite dimensional $k$-algebra, then so is $B$.

Proposition 3.2 Let $B$ be an excellent extension of a finite dimension 
$k$-algebra $A$, then $B$ is of tame type if and only if so is $A$.

Proof. Our proof is based on the definition of a tame algebra.

We first show the sufficiency of the condition. Let $B \geq A$ be an excellent extension and let $A$ be a tame algebra. By Lemma 2.3, $B$ is not of finite representation type. Let $d$ be a positive integer and $X$ an indecomposable right $B$-module with $\operatorname{dim}_{k} X=d$. By Lemma 2.4, there is an indecomposable direct summand $Y_{A}$ of $X_{A}$ such that $X_{B} \mid\left(Y \otimes_{A} B\right)_{B}$. Hence $\operatorname{dim}_{k} Y \leq d$.

Since that $A$ is a tame algebra, there are a finite number of $k[T]-A$ bimodules $\left\{M_{j}: j=1, \cdots m\right\}$, which are free as left $k[T]$-modules such that all but a finite number of indecomposable $A$-modules of dimension not bigger than $d$ are isomorphic to $k[T] /(T-\lambda) \otimes_{k[T]} M_{j}$ for some $\lambda \in k$. No loss of generality, we may assume that $Y_{A} \cong k[T] /(T-\lambda) \otimes_{k[T]} M_{t}$ for some $t$ and some $\lambda \in k$. It is easy to see that $M_{t} \otimes_{A} B$ is free as a left $k[T]$-module from the same property of $\left(M_{t}\right)_{A}$, because $B$ is a finite free left $A$-module. Hence $M_{t} \otimes_{A} B \in G C(B)$. Thus we have $M_{t} \otimes_{A} B=\oplus_{s=1}^{m_{t}} M_{t}^{s}$ for some positive integer $m_{t}$, where each $M_{t}^{s}$ is indecomposable in $G C(B)$. By Lemma 2.5, $k[T] /(T-\lambda) \otimes_{k[T]} M_{t}^{s}$ is indecomposable as an $k[T] /(T-\lambda)$ - $B$-bimodule. That is, $k[T] /(T-\lambda) \otimes_{k[T]} M_{t}^{s}$ is an indecomposable right $B$-module because $k[T] /(T-\lambda) \cong k$ as algebras.

Notice that $Y \otimes_{A} B \cong\left(k[T] /(T-\lambda) \otimes_{k[T]} M_{t}\right) \otimes_{A} B \cong \oplus_{s=1}^{m_{t}}\left(\left(k[T] /(T-\lambda) \otimes_{k[T]}\right.\right.$ $\left.M_{t}^{s}\right)$. We have $X_{B} \cong k[T] /(T-\lambda) \otimes_{k[T]} M_{t}^{i}$ for some $i$. Because the set $\left\{M_{j}^{i}\right.$ : $\left.j=1, \cdots m ; i=1, \cdots, m_{j}\right\}$ is finite, $B$ is a tame algebra.

Conversely, assume that $B$ is a tame algebra. Then $A$ is not of finite representation type by Lemma 2.3 again. Let $d$ be a positive integer and $X^{\prime}$ an indecomposable right $A$-module with $\operatorname{dim}_{k} X^{\prime}=d$, there is an indecomposable direct summand $Y_{B}^{\prime}$ of $X^{\prime} \otimes_{A} B$ such that $X_{A}^{\prime} \mid Y_{A}^{\prime}$.

Notice that $B$ is a tame algebra, there are a finite number of $k[T]-B$ bimodules $\left\{N_{j}: j=1,2, \cdots, n\right\}$, which are free as left $k[T]$-modules such that all but a finite number of indecomposable $B$-modules of dimension not bigger than $d$ are isomorphic to $k[T] /(T-\lambda) \otimes_{k[T]} N_{j}$ for some $j$ and some $\lambda \in k$. To prove that $A$ is a tame algebra, it is no harm to assuming that $Y_{B}^{\prime} \cong k[T] /(T$ $\lambda) \otimes_{k[T]}\left(N_{t}\right)_{B}$ for some $t$ and some $\lambda \in k$. It is easy to see that $\left(N_{t}\right)_{A}$ is free as a left $k[T]$-module from the same property of $\left(N_{t}\right)_{B}$. Hence, $\left(N_{t}\right)_{A} \in G C(A)$. We have $\left(N_{t}\right)_{A}=\oplus_{s=1}^{m_{t}} N_{t}^{s}$ for some positive integer $m_{t}$, where each $N_{t}^{s}$ are indecomposable in $G C(A)$. By Lemma 2.5, $X_{A}^{\prime} \cong k[T] /(T-\lambda) \otimes_{k[T]} N_{t}^{s}$ is indecomposable as an $k[T] /(T-\lambda)$-A-bimodule. It is equivalent to saying that $X_{A}^{\prime} \cong k[T] /(T-\lambda) \otimes_{k[T]} N_{t}^{s}$ is an indecomposable $A$-module because $k[T] /(T$ - 
$\lambda) \cong k$ as algebras. Since the set $\left\{N_{j}^{i}: j=1, \cdots, n ; i=1, \cdots, ; m_{j}\right\}$ are finite, and so $A$ is a tame algebra.

According to the Drozd's tame-and-wild theorem and Lemma 2.3 and Proposition 3.1, we obtain our main result.

Theorem 3.3 Let $B$ be an excellent extension of $A$, then $A$ and $B$ have the same representation type.

Acknowledgments: This research was partially supported by NSFC (Grant Nos. 11326065, 11201220), and the Key Project of He nan Educational Committee (Grant No. 13A110834).

\section{References}

[1] L.Bonami, On the Structure of Skew Group Rings, Algebra Berichte 48, Verlag Reinhard Fischer, Munichen, 1984.

[2] W.W. Crawley-Boevey, Tame Algebras and Generic Modules, Proceedings of the London Mathematical Society, 63(1991),241-264.

[3] W.W. Crawley-Boevey, On tame algebras and bocses, Proceedings London Mathematical Society, 5(1998), 451-483.

[4] Y.A. Drozd, Tame and wild matrix problems, representations and quadratic forms, American Mathematical Society Translations, 128(1986), 31-55.

[5] L.G. Feng, Essential extensions, excellent extensions and finitely presented dimension, Acta Mathematica Sinica(English Series), 13(1997), 231-238.

[6] Z.Y. Huang, and J.X. Sun, Invariant properties of representations under excellent extensions, Journal of Algebra, 358(2012), 87-101.

[7] S. Kasjan, Base field extensions and generic modules over finite dimensional algebras, Archiv der Mathematik, 77, no. 2, 155-162.

[8] G.X. Liu,, On the structure of tame graded basic Hopf algebras, Journal of Algebra, 299(2006), 841-853.

[9] D.S. Passman, The Algebraic Structure of Group Rings, WileyInterscience, New York London-Sydney, 1977. 
[10] M.M. Parmenter, and P.N. Stewart, Excellent extensions, Communication in Algebra, 16(1988), 703-713.

[11] W.M. Xue, On almost excellent extensions, Algebra Colloquium, $3(1996), 125-134$.

[12] Xiao, Y.F., SF-rings and excellent extensions, Communication in Algebra, 22(1994), 2463-2471.

Received: March 11, 2014 